\title{
P01-1-28 Poster session
}

\section{In response to monomeric $\alpha$-synuclein, brain pericytes release inflammatory cytokines to impair brain endothelial barrier}

\author{
Shinya Dohgu, Fuyuko Takata, Junichi Matsumoto, Ikuya Kimura, Atsushi Yamauchi, \\ Yasufumi Kataoka
}

Department of Pharmaceutical Care and Health Sciences, Fukuoka University, Japan

Background. Blood-brain barrier (BBB) is composed of brain endothelial cells, pericytes, and astrocytes to regulate brain homeostasis. Its disruption occurs in the process of various neurodegenerative diseases including Parkinson disease (PD). $\mathrm{PD}$ is characterized by aggregated $\alpha$-synuclein and loss of dopaminergic neurons in substantia nigra. Therefore, $\alpha$ synuclein could be involved in BBB disruption in patients with PD. Here, we investigated the ability of $\alpha$-synuclein to induce the release of inflammatory cytokines from each BBB comprising cell type in relation to an impairment of the BBB. Methods. Primary cultures of rat brain endothelial cells (RBECs) and rat brain pericytes are isolated from 3-week-old Wistar rats. Using Transwell inserts, we made two types of the in vitro BBB models; one using RBECs alone (RBEC monolayer) and the other containing brain pericytes (RBEC/Pericyte coculture). These models were exposed to recombinant human $\alpha$-synuclein (up to $50 \mu \mathrm{g} / \mathrm{mL}$ ) for $24 \mathrm{hr}$ by adding it to the luminal or abluminal chambers of the in vitro BBB model. Then, the transendothelial electrical resistance and the permeability coefficient of sodium fluorescein (Na-F) to RBECs were measured. Release of cytokines into culture supernatants were determined by ELISA.

Results. In RBEC/pericyte coculture, $\alpha$-synuclein added to the abluminal side increased Na-F permeability coefficient and decreased TEER. While it had no effect, when added to the luminal side. In RBEC monolayer, $\alpha$-synuclein failed to affect $\mathrm{Na}-\mathrm{F}$ permeability when added to the luminal or abluminal side. Brain pericytes but not RBECs released various inflammatory cytokines/chemokines (IL-1 $\beta$, IL-6, MCP-1, and TNF- $\alpha$ ) and matrix metalloproteinase-9 after an exposure of $\alpha$-synuclein for 24 h. $\alpha$-Synuclein was not self-assembled during 24 -h exposure and it was taken up by RBECs and pericytes.

Conclusion. Brain pericytes are more sensitive to monomeric $\alpha$-synuclein than brain endothelial cells in terms of the release of various inflammatory cytokines/chemokines and MMP-9. These pericyte-derived factors could contribute to BBB breakdown in patient with PD. 